\title{
Immune reconstitution inflammatory syndrome associated with acquired immunodeficiency syndrome-related gastrointestinal limited Kaposi's sarcoma presenting as acute intestinal obstruction: a case report
}

\author{
Jyotirmoy Pal ${ }^{1}$, Ankit Shrivastav ${ }^{2 *}$, Hari Shankar Pathak ${ }^{3}$ and Dipendra Kumar Sarkar ${ }^{4}$
}

\begin{abstract}
Introduction: Immune reconstitution inflammatory syndrome during anti-retroviral treatment of acquired immunodeficiency syndrome (AIDS) -associated gastrointestinal Kaposi's sarcoma has rarely been reported.

Case Presentation: A 36-year-old Asian Indian male, newly diagnosed with AIDS and treatment naïve, was started on highly active antiretroviral therapy (HAART). He developed acute intestinal obstruction after four weeks of therapy. A laparotomy was done with excision and adhesiolysis leading to relief of symptoms. A histology report revealed the lesion to be Kaposi's sarcoma. Our patient was diagnosed to be having immune reconstitution inflammatory syndrome associated with AIDS-associated gastrointestinal limited Kaposi's sarcoma, which presented as acute intestinal obstruction. Our patient was treated with paclitaxel post-operatively and HAART was continued. Our patient responded to therapy.

Conclusion: Immune reconstitution inflammatory syndrome involving Kaposi's sarcoma may occur in HAART-naïve individuals with AIDS-related Kaposi's sarcoma. Gastrointestinal Kaposi's sarcoma may present with sudden increase in size or inflammation leading to acute intestinal obstruction. This does not indicate failure of HAART or a need for changes in anti-retroviral regimen.
\end{abstract}

\section{Introduction}

Kaposi's sarcoma (KS) remains the most common tumor in individuals infected with human immunodeficiency virus (HIV) and is associated with significant morbidity and mortality. Highly active anti-retroviral therapy (HAART) for acquired immunodeficiency syndrome (AIDS) decreases the incidence of KS, prolongs the time to treatment failure in KS, leads to resolution of individual lesions, and also decreases KS herpes virus viral load. Immune reconstitution inflammatory syndrome (IRIS) is well recognized as a complication of using HAART for treating AIDS, especially when associated with mycobacterial, fungal or viral infection. Our case

\footnotetext{
* Correspondence: ankit.med@gmail.com

${ }^{2} \mathrm{RMO}$ cum Clinical Tutor, Department of Medicine, Institute of Postgraduate Medical Education \& Research, Kolkata, India

Full list of author information is available at the end of the article
}

demonstrates that KS can worsen during HAART-associated increases in the cluster of differentiation 4 (CD4) count and KS is an IRIS-associated disease. IRIS-KS may be associated with significant complications. It is important for clinicians to realize that KS-associated IRIS does not indicate failure of HAART or a need for changes in the anti-retroviral regimen. Instead, chemotherapy in conjunction with HAART can effectively control the symptoms of IRIS as well as resolve KS.

\section{Case presentation}

A 36-year-old Asian Indian male patient attended our clinical immunology outpatient clinic with a fever of two months duration and two episode of herpes zoster in the last year. He also complained of significant weight loss in the last three months. He had no history of chronic cough or diarrhea. He was not a diabetic and 
not alcoholic. Our patient had a history of multiple unprotected sexual exposure around eight years ago.

On clinical examination his vitals were normal. $\mathrm{He}$ had a mild pallor and oral candidiasis. He had no significant lymphadenopathy or any cutaneous lesions. A systemic examination revealed no significant abnormality. Routine investigations were within normal limits. An ultrasound of his abdomen revealed no abnormality. An enzyme-linked immunosorbent assay (ELISA) for HIV-1 was positive and this was confirmed by western blot. Serology for Hepatitis B and C was non-reactive. Our patient's CD4 count was $67 / \mathrm{mm}^{3}$. He was put on HAART therapy consisting of nevirapine $30 \mathrm{mg}$ twice a day, lamuvidine $150 \mathrm{mg}$ twice a day and zidovudine 300 mg twice a day.

After 12 days our patient came back with a complaint of upper abdominal discomfort. An abdominal examination revealed mild epigastric tenderness, no organomegaly and normal peristaltic sound. An ultrasound of his abdomen was normal. He was presumed to be suffering from gastrointestinal side-effects, which is very common during initiation of HAART, and advised to take a proton pump inhibitor.

Our patient came back after another two weeks with severe abdominal pain, vomiting and abdominal distension. On examination his abdomen was found to be distended with absent peristaltic sound. An ultrasound of his abdomen was done and showed distended bowel loops with an admixture of air and fluid suggestive of acute intestinal obstruction. Routine investigations were normal and his CD4 count was $356 / \mathrm{mm}^{3}$. We put our patient on conservative management with fluids and nasogastric suction. However there was no improvement in 24 hours and our patient complained of increasing pain. A surgical consultation was taken and an exploratory laparotomy was performed with universal precautions. On exploration, small bowel loops were seen matted with a minimal amount of ascites. Purple colored patches, three in number, were seen on the serosal surface of his small bowel. Three thick walled, purple colored rounded cystic lesions adherent to surrounding bowel loops and omentum containing dirty white fluids were seen on the small bowel mesentery. The lesions had prominent veins over their surfaces (Figures 1 and 2). The lesions were excised; adhesiolysis was performed followed by peritoneal lavage with normal saline. The excised lesion was sent for histopathology.

Our patient's symptoms improved after surgery. $\mathrm{He}$ was started on an oral diet after five days. The histological examination of the excised lesion showed tortuous blood vessels lined by a single layer of pleomorphic endothelial cells with extra-vasation of red blood cells in the stroma with spindle cells (Figure 3) suggestive of

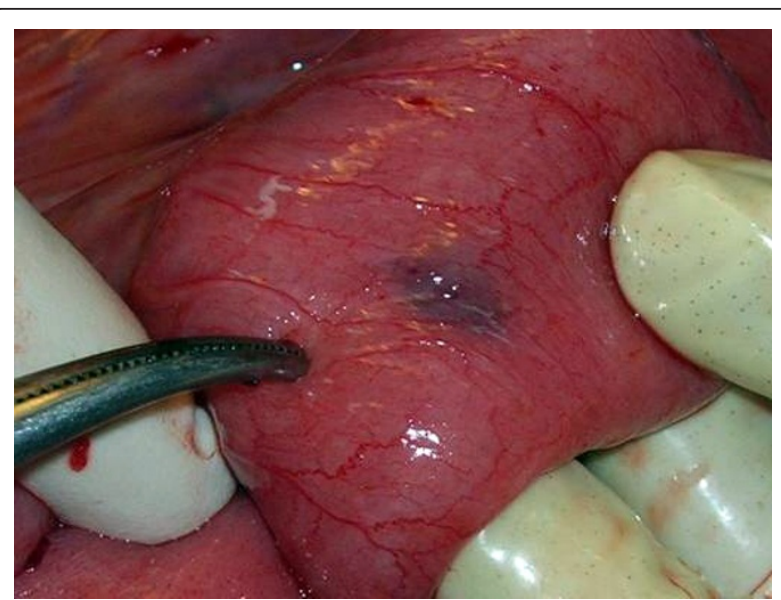

Figure 1 Purple colored patches on the small bowel serosal surface seen during laparotomy.

KS. His CD4 count was repeated and was $476 / \mathrm{mm}^{3}$. Our patient was started on paclitaxel (eight cycles of $100 \mathrm{mg} / \mathrm{m}^{2}$ intravenously over three hours every two weeks) and the HAART was continued along with supportive management. He responded well to treatment and is doing well on follow-up.

\section{Discussion}

KS is a multi-focal neoplastic disease that originates from the lymphatic endothelium, most frequently involving the skin. Also commonly involved are the mucous membrane, lymph nodes, gastrointestinal system and lungs. Lesions have been reported in virtually every organ including the heart and central nervous system. Human herpes virus- 8 has been strongly implicated as a co-factor in the pathogenesis of KS. In HIV-infected patients, KS is an AIDS-defining illness.

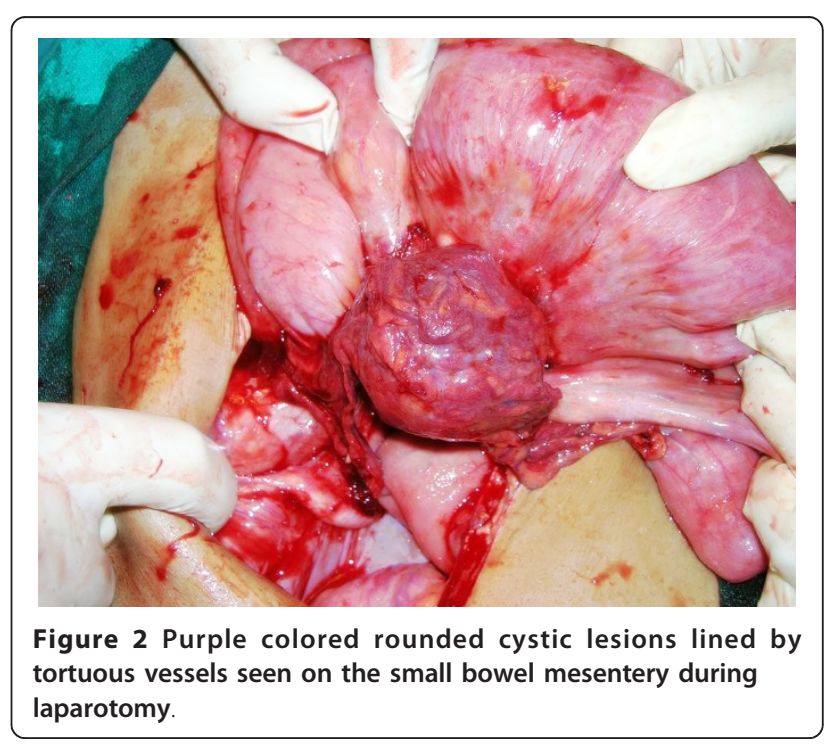




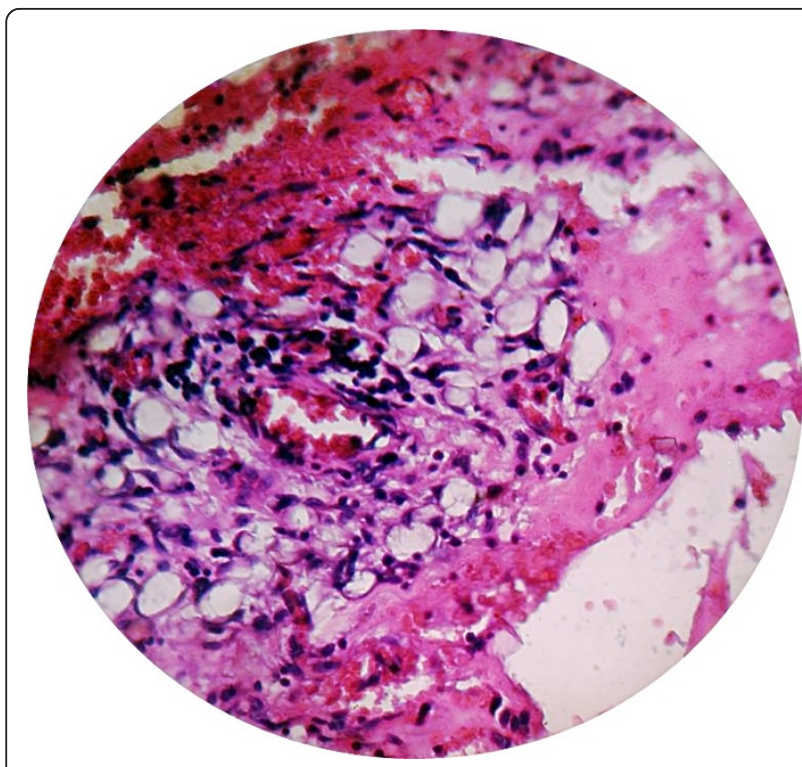

Figure 3 Biopsy of excised lesion showing tortuous blood vessels, proliferation of pleomorphic endothelial cells with extra-vasation of red blood cells in stroma. (H\&E stain, 400X).

Gastrointestinal involvement in $\mathrm{KS}$ is frequent in patients with advanced HIV disease. In one series of KS, gastrointestinal lesions were found in up to $51 \%$ of the cases [1]. Most clinical series have underestimated the overall incidence of luminal gastrointestinal involvement with KS because intestinal lesions rarely lead to symptoms. In a prospective endoscopic evaluation of 50 AIDS patients with KS, gastrointestinal tract involvement was present in almost all cases [2]. In another series, gastrointestinal involvement was reported in $40 \%$ of cases at initial diagnosis and up to $80 \%$ at autopsy.

Gastrointestinal involvement commonly occurs in association with cutaneous lesions or lymph node involvement, with gastrointestinal tract involvement alone occurring in only $3.5 \%$ of cases [3]. The absence of skin or lymph node KS, however, does not exclude the possibility of gastrointestinal involvement [2]. Gastrointestinal $\mathrm{KS}$ is mostly found in the stomach and duodenum with jejunum, ileum or large bowel rarely being involved. The biliary tract is also commonly involved. Lesions appear either as macule, sub-mucosal growth or as nodules [2]. Most of the lesions (80\%) are clinically silent with bleeding, protein-losing enteropathy, malabsorption and obstructive jaundice being the most common presentation. Gastrointestinal obstruction has also been rarely reported [2].

IRIS is an inflammatory reaction to an opportunistic pathogen and/or tumor antigen that occurs early after initiation of HAART in patients with AIDS and is temporally related to an increase in the host's CD4+ lymphocyte count [4]. IRIS is most frequently observed in individuals with severe CD4+ T-cell depletion and is believed to be due to reconstitution of immune responses to a previously existing (but clinically occult or previously treated) pathogen or tumor antigen, rather than development of a new opportunistic infection or progression of opportunistic infection due to treatment failure. Our patient had AIDS and started on HAART therapy. He was admitted with severe pain abdomen after four weeks of HAART and diagnosed to be having intestinal obstruction. An emergency laparotomy was done which showed matted small bowel loops with purple colored patches and cysts with adhesions on the small bowel serosal surface and mesentery. Adhesiolysis and resection were done and the lesion sent for histopathological examination, which showed it to be a case of KS. Our patient had no features of KS during initiation of HAART. An ultrasound of his abdomen was also normal.

Initiation of HAART is usually associated with a regression of KS. However in this case there was probably a rapid increase in the size of the KS lesion, causing intestinal obstruction. Several features of this case suggest that the worsening symptoms and clinical finding represented IRIS rather than progressive KS. The rise in his CD4 count and the temporal relationship of bowel obstruction to HAART initiation also support the diagnosis of KS-IRIS.

Although KS is prevalent among HIV-1 infected persons, IRIS during anti-retroviral treatment of AIDSassociated KS has only been reported three times [5-7]. In one case, laryngeal obstruction occurred in a patient with known KS shortly after initiation of HAART [5]. In the second case, parotid gland KS developed in an individual two years after initiation of HAART, despite there being good CD4+ lymphocyte reconstitution and virus suppression [6]. In the third case, rapidly progressive KS lesions with lymphadenopathy and tissue swelling occurred in a patient during anti-retroviral treatment, despite an increased CD4+ lymphocyte count and decreased HIV-1 level and KS-associated herpes virus replication [8].

In a review of 5,832 patients with AIDS undergoing HAART, Bower et al. identified 150 therapy-naïve patients with a first presentation of $\mathrm{KS}$ and recorded their clinic-pathologic features prospectively[9]. They identified ten patients with IRIS-KS in the patient cohort of HIV patients with KS who were started on HAART.

This is a rare case of IRIS associated with AIDSrelated gastrointestinal limited $\mathrm{KS}$, presenting as an acute intestinal obstruction. It is likely that KS-associated IRIS is more common than the literature reflects due to limited awareness of this condition. It is important for clinicians to realize that KS-associated IRIS does 
not indicate failure of HAART or a need for changes in the anti-retroviral regimen. Instead, chemotherapy in conjunction with HAART can effectively control the symptoms of IRIS as well as resolve KS, especially when KS-IRIS is severe or there is visceral involvement.

\section{Conclusions}

IRIS-KS may occur in HAAR- naïve individuals with AIDS-related KS. Gastrointestinal KS may present with a sudden increase in size leading to acute intestinal obstruction. KS-associated IRIS does not indicate failure of HAART or a need for changes in the anti-retroviral regimen.

\section{Consent}

Written informed consent was obtained from the patient for publication of this case report and any accompanying images. A copy of the written consent is available for review by the Editor-in-Chief of this journal

\section{Author details}

${ }^{1}$ Associate Professor, Department of Medicine, Institute of Postgraduate Medical Education \& Research, Kolkata, West Bengal, India. ${ }^{2}$ RMO cum Clinical Tutor, Department of Medicine, Institute of Postgraduate Medical Education \& Research, Kolkata, India. ${ }^{3}$ Associate Professor, Department of Medicine, KPC Medical College \& Hospital, Kolkata, India. ${ }^{4}$ Associate Professor, Department of Surgery, Institute of Postgraduate Medical Education \& Research, Kolkata, India.

\section{Authors' contributions}

$\mathrm{JP}$ and AS were involved in patient care and management. They also conceived the report and were involved in preparation of the manuscript. DKS was involved in the surgery and patient management. HSK helped diagnose our patient and review the manuscript. DG was involved in counseling and administration. All authors read and reviewed the manuscript.

\section{Competing interests}

The authors declare that they have no competing interests.

Received: 21 March 2010 Accepted: 26 July 2011

Published: 26 July 2011

\section{References}

1. Parente F, Cernuschi M, Orlando G, Rizzardini G, Lazzarin A, Bianchi Poro G: Kaposi's sarcoma and AIDS: frequency of gastrointestinal involvement and its effect on survival: a prospective study in a heterogeneous population. Scand J Gastroenterol 1991, 26(10):1007-1012.

2. Friedman S, Wright T, Altman S: Gastrointestinal Kaposi's sarcoma in patients with acquired immunodeficiency syndrome: endoscopic and autopsy finding. Gastroenterology 1985, 89(1):102-108.

3. Heiss W, Mostertz P, Skorde J, Nehm K, Grosse G, L'age M: Gastrointestinal Kaposi sarcoma in AIDS - endoscopic findings [abstract]. International Conference on AIDS, 4-9 June 1989 1989, 349.

4. Hirsch HH, Kaufmann G, Sendi P, Battegay M: Immune reconstitution in HIV-infected patients. Clin Infect Dis 2004, 38(8):1159-1166.

5. Weir A, Wansbrough-Jones M: Mucosal Kaposi's sarcoma following protease inhibitor therapy in an HIV-infected patient. AIDS 1997, 11(15):1895-1896.

6. Rizos E, Drosos AA, loannidis JP: Isolated intraparotid Kaposi sarcoma in human immunodeficiency virus type 1 infection. Mayo Clin Proc 2003, 78(12):1561-1563.
7. Leidner RS, Aboulfia DM: Recrudescent Kaposi's sarcoma after initiation of HAART: a manifestation of immune reconstitution syndrome. AIDS Patient care STDS 2005, 19(10):849-856.

8. Connick E, Kana MA, White IE, Ryder J, Campbell TB: Immune reconstitution inflammatory syndrome associated with Kaposi sarcoma during potent antiretroviral. Clin Infect Dis 2004, 39(12):1852-1855.

9. Bower $M$, Nelson $M$, Young $M$, Thirlwell C: Immune reconstitution inflammatory syndrome associated with Kaposi's sarcoma. J Clin Oncol 2005, 23(22):5224-5228.

doi:10.1186/1752-1947-5-327

Cite this article as: $\mathrm{Pal}$ et al: Immune reconstitution inflammatory syndrome associated with acquired immunodeficiency syndromerelated gastrointestinal limited Kaposi's sarcoma presenting as acute intestinal obstruction: a case report. Journal of Medical Case Reports 2011 5:327.

\section{Submit your next manuscript to BioMed Central and take full advantage of:}

- Convenient online submission

- Thorough peer review

- No space constraints or color figure charges

- Immediate publication on acceptance

- Inclusion in PubMed, CAS, Scopus and Google Scholar

- Research which is freely available for redistribution 